\title{
Morfogeometría del caparazón del cangrejo Damithrax spinosissimus (Decapoda: Mithracidae) en tres islas del Caribe suroccidental
}

\author{
Bibian Martínez-Campos ${ }^{1}$, Néstor Hernando Campos $^{2}$, Adriana Bermúdez Tobón ${ }^{3}$ \& \\ Edna Judith Márquez ${ }^{4}$ \\ 1. Posgrado en Ciencias, Biología, Línea de Biología Marina, Universidad Nacional de Colombia Sede Caribe. Calle 25 \\ No. 2-55, Playa Salguero, Santa Marta D.T.C.H., Colombia; usagiblacklady@gmail.com \\ 2. CECIMAR, Universidad Nacional de Colombia Sede Caribe. Calle 25 No. 10 2-55, Playa Salguero, Santa Marta \\ D.T.C.H., Colombia; nhcamposc@unal.edu.co \\ 3. Facultad de Ciencias Exactas y Naturales. Universidad de Cartagena. Avenida 13 del Consulado \# Calle 30 No. \\ 48-152, Cartagena, Bolívar, Colombia; abermudezt@unicartagena.edu.co \\ 4. Universidad Nacional de Colombia-Sede Medellín-Facultad de Ciencias-17 Escuela de Biociencias-Laboratorio de \\ Biología Celular y Molecular, Calle 59A No 63 - 2018 Bloque 19 A Laboratorio 310, Medellín, 050034, Colombia; \\ ejmarque@unal.edu.co,ejmarque@gmail.com
}

Recibido 11-V-2015. Corregido 05-I-2016. Aceptado 29-I-2016.

\begin{abstract}
Morfogeometry shell crab Damithrax spinosissimus (Decapoda: Mithracidae) on three islands in the southwestern Caribbean. The Caribbean King Crab, Damithrax spinosissimus is a fishery resource, but few biological studies are available. Its patchy distribution, and the high environmental heterogeneity due to the oceanographic, landscape, and ecological characteristics of the Caribbean Sea, can favor the phenotypic variability according to the geographic origin. For this reason, the objective of our study was to determine morphometic variability in the carapace of the crabs from three Southwestern Caribbean islands: Providence, Rosario and San Bernardo. The former has an oceanic influence, whereas the two latter islands, which are closer to the mainland, have more influence of the coastal dynamic. A total of 276 individuals from the three islands were captured and photographed: 103 females and 173 males; their variation was analyzed from 12 anatomical landmarks marked on one half of the carapace. The differences in the carapace size were calculated using a Fisher's pairwise comparison; the allometric effect was calculated by Multivariate Regression Analysis; and the Allometric model via Multivariate Analysis of Covariance. The average shape was calculated from the relative wraps RW obtained through PCA analysis; and the Euclidian distances between the shape averages, were used to construct a tree using the Neighbour-Joining algorithm over 10000 permutations. The results showed significant differences in the size and shape of the carapace between sexes and among the three islands. The differences in the shape of the crabs from Providencia were significantly greater than those found between the crabs of Rosario and San Bernardo. These results can be explained inclusively due to the genetic differences and phenotypic plasticity, due to environmental heterogeneity of the sector. This study, the first of its kind, is a contribution to the knowledge of the species. We concluded that different management strategies should be differently adopted in each of the three studied islands. Rev. Biol. Trop. 64 (2): 667-681. Epub 2016 June 01.
\end{abstract}

Key words: morfogeometrics, phenotypic plasticity, vulnerable specie, Southwestern Caribbean, crustaceans.

El "cangrejo rey" Damithrax spinosissimus (Lamark, 1818) pertenece a la familia Mithracidae y recientemente fue reasignado del género Mithrax al nuevo género Damithrax propuesto por Windsor y Felder (2014). Por su tamaño y palatabilidad es un recurso pesquero del mar Caribe; se caracteriza por una coloración rojo carmín brillante o vino, presenta un caparazón subtriangular, un poco más ancho que largo, con un rostro pequeño formado por dos cuernos cortos truncados y las dos primeras espinas del borde anterolateral bífidas. Los quelípedos presentan un marcado dimorfismo, siendo más robustos en los machos (Williams, 1984) y por 
esta razón son más apetecidos para el consumo humano que las hembras.

D. spinosissimus exhibe una distribución amplia registrándose en Carolina del Sur, La Florida, las Antillas, Venezuela, Golfo de México, Nicaragua, Panamá y Colombia (Williams, 1984; Felder, Álvarez, Goy, \& Lemaitre, 2009). En contraste con su amplia distribución, presenta un patrón agregado debido a la especificidad de su hábitat, restringiéndose a parches arrecifales que constituyen agrupaciones parcialmente aisladas (Bermúdez, Campos, \& Navas, 2002). Este tipo de distribución en mosaico se ha estudiado en varias especies de crustáceos en el mar Caribe colombiano y se ha demostrado que propician patrones de distribución bien definidos, endemismos y variabilidad fenotípica intraespecífica (Bermúdez, 2007; Martínez-Campos, Campos, \& Bermúdez, 2012).

Las agrupaciones de $D$. spinosissimus pueden estar adicionalmente influenciadas por una baja dispersión de larvas debido a la corta duración del período larvario, que dura cinco o seis días (Provenzano \& Bronwell, 1977; Boltovskoy, 1981). Lo anterior es relevante para el Caribe suroccidental, ya que la región se caracteriza por una circulación a mesoescala dominada por remolinos y giros ciclónicos (Andrade, 2001; Andrade, Barton, \& Mooers, 2003; Lin, Sheng, \& Greatbatch, 2012). Dicho efecto presenta tal magnitud que afecta la distribución de las larvas, por lo que se ha considerado al suroccidente del Caribe como la región con las tasas más elevadas de retención (57\%); haciendo que el intercambio incluso entre regiones cercanas, no sea suficiente para mantener el flujo poblacional de varias especies (Cowen, Paris, \& Srinivasan, 2006).

Actualmente, D. spinosissimus se encuentra catalogado en el libro rojo de invertebrados marinos de Colombia como vulnerable, ya que es apetecido como recurso pesquero y su abundancia es escasa (Bermúdez et al., 2002). Sin embargo, hasta el momento no existen estudios que pormenoricen su estado a nivel local; no obstante, se piensa que puede existir diferenciación fenotípica debido a que los adultos por lo general, tienen una distribución local restringida hacia las áreas insulares. Determinar si existen diferencias morfológicas es relevante para establecer si los individuos están sometidos a los mismos procesos de adaptación local a pesar de su distribución en parches agregados; o bien, para establecer si existe algún tipo de variabilidad.

La morfometría geométrica basada en puntos anatómicos de referencia, es una herramienta que permite establecer dichas diferencias y aventaja a la morfometría tradicional al ser un método matemático, que permite detectar el cambio de la forma de los organismos y la identificación de grupos dentro de las especies, brindando un tratamiento estadístico a los caracteres geométricos de las formas biológicas (Cadrin, 2000). Este método se ha utilizado para estudiar varias estructuras anatómicas en crustáceos, incluyendo el caparazón, en especies como Liocarcinus depuratus (Rufino, Abelló, \& Yule, 2004), Petrolisthes galathinus (Hiller, Kraus, Almon, \& Werding, 2006), Agononida longipes, Munida flinti, Munidopsis riveroi (Bermúdez, 2007), Carcinus maenas (Ledesma, Van der Molen, \& Barón, 2010; Silva, Alves, Paula, \& Hawkins, 2010), Aegla marginata (Trevisan, Zanetti-Marochi, Costa, Santos, \& Masunari, 2012), Cyrtograpsus affinis (Lezcano, González-José, Spivak, \& Dellatorre, 2012) y Cyrtograpsus angulatus (Idaszkin, Márquez, \& Nocera, 2014), entre otros.

El objetivo de este estudio fue determinar la posible variabilidad intraespecífica de $D$. spinosissimus en función de su procedencia geográfica dada una distribución agregada, para lo cual se estudió la variación morfométrica del caparazón de individuos colectados en tres islas del Caribe suroccidental: Providencia, Rosario y San Bernardo. La primera de ellas notablemente apartada del continente, con una influencia netamente oceánica, con temperaturas y niveles de salinidad uniformes (CORALINA-INVEMAR, 2012) y las dos siguientes cercanas al continente, influenciadas por sus procesos geomorfológicos y por la fuerte dinámica costera, que en el suroccidente 
del Caribe colombiano se debe principalmente a los ríos Sinú y Magdalena (Posada, Morales, \& Henao, 2011).

\section{MATERIALES Y MÉTODOS}

Área de estudio: El mar Caribe es un sistema semicerrado perteneciente al océano
Atlántico occidental tropical (Fig. 1a), conformado por cinco cuencas: Granada, Venezuela, Colombia, Caimán y Yucatán. La cuenca Colombia limita al suroccidente con el continente, incluyendo a Panamá, Nicaragua, Honduras y parte del continente colombiano y al norte con Haití, República Dominicana y Jamaica. La dorsal de la Beata la separa de la
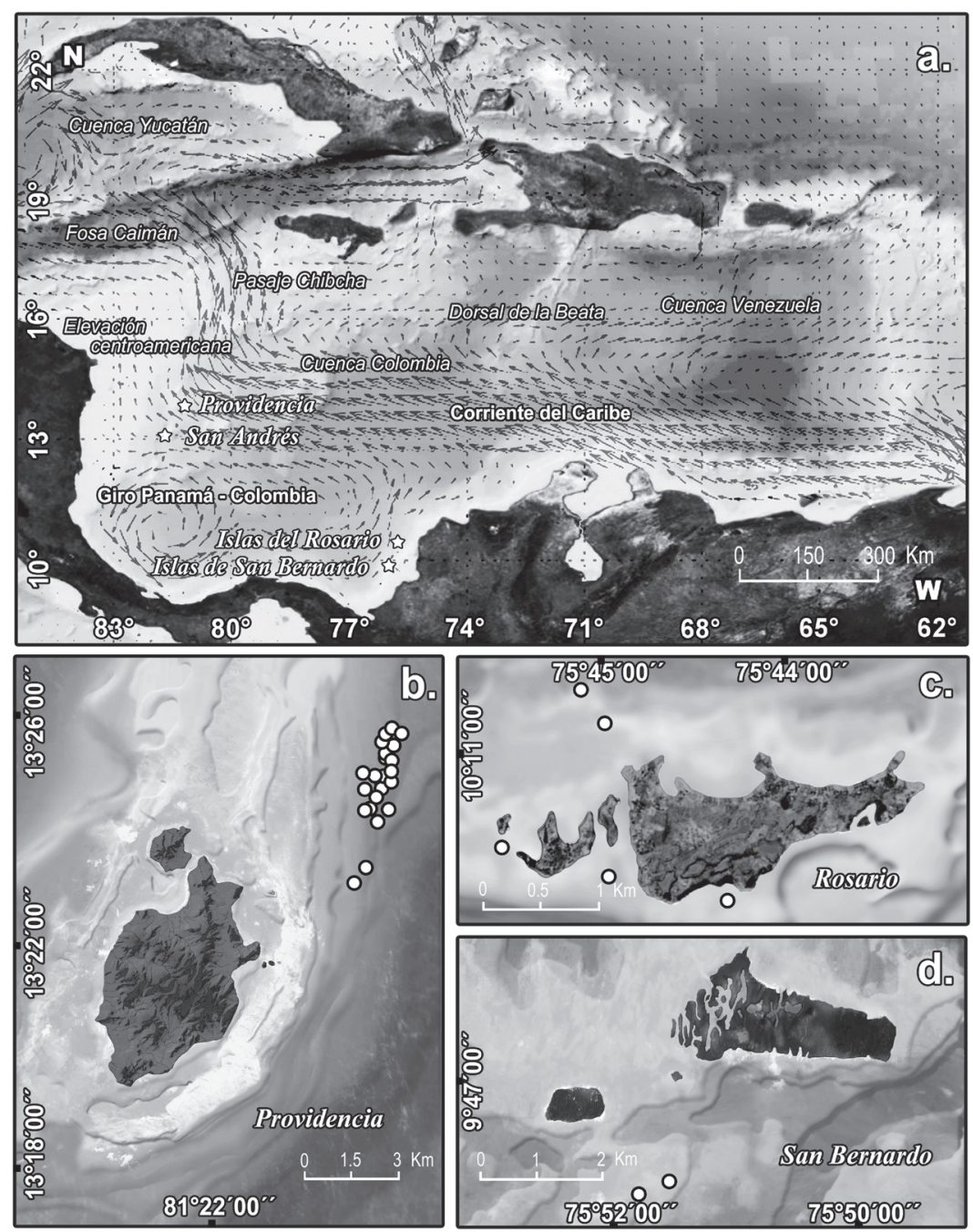

Fig. 1. a. Mar Caribe, variación geomorfológica y dinámica media superficial de las corrientes obtenida a partir de los resultados de velocidad [cm s-1] del Parallel Oceanic Circulation Model; la dinámica de las corrientes está basada en Ruíz-Ochoa (2011). b. Providencia. c. Rosario. d. San Bernardo (Tomado de la base cartográfica SIG del INVEMAR). Los círculos blancos indican las estaciones de muestreo.

Fig. 1. a. Caribbean Sea including the geomorphologic variation and superficial average dynamic of currents obtained from velocity results of the Parallel Oceanic Circulation Model. The dynamics of the currents are modified from Ruíz-Ochoa (2011) b. Providence. c. Rosario. d. San Bernardo (Taken from the Cartographic base SIG INVEMAR). The white circles indicate the sampling sites. 
cuenca de Venezuela y la elevación centroamericana la separa de la cuenca Caimán; las cuencas Colombia y Caimán se conectan mediante el canal Chibcha (Fig. 1a), por el cual, circula casi el $60 \%$ de la masa de agua de la corriente del Caribe (Andrade, 2001).

El archipiélago de "San Andrés, Providencia y Santa Catalina", se conforma por atolones dispuestos a lo largo de fracturas tectónicas y es uno de los sistemas arrecifales más extensos del Atlántico y el de mayor extensión de Colombia, por lo que está catalogado como reserva de la biosfera (CORALINA-INVEMAR, 2012). Providencia está ubicada en $13^{\circ} 19^{\prime} 11^{\prime}$ '

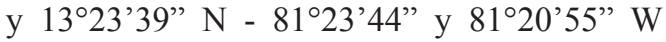
(Fig. 1b) y se formó a partir un volcán andesítico extinto. El complejo moderno de arrecifes de barrera, borde, franja, pináculos y parches lagunares, así como las playas y manglares se formaron en la última transgresión del Holoceno (Díaz et al., 2000; Posada et al., 2011).

El desarrollo de estructuras arrecifales con andamiajes calcáreos bien consolidados, es una de las principales características que diferencia a Providencia de las islas cercanas al continente, propiciando la diversidad de la fauna coralina que incluye más de 50 especies de corales pétreos, unas 40 de octocorales, diversas algas calcáreas y más de 100 especies de esponjas. Por su parte, los arrecifes de las islas cercanas al continente son en su mayoría de franja o de plataforma, localizados principalmente en ensenadas y bahías formando pequeños archipiélagos cerca de la costa (Díaz et al., 2000; Posada et al., 2011).

Las islas del Rosario $\left(10^{\circ} 11^{\prime} 49^{\prime \prime}\right.$ y $10^{\circ} 08^{\prime} 38^{\prime \prime} \mathrm{N}-75^{\circ} 47^{\prime} 45^{\prime \prime}$ у 7 75॰43'14” W), y el archipiélago de San Bernardo (9 $9^{\circ} 43^{\prime} 54^{\prime \prime}$ y $9^{\circ} 48^{\prime} 20^{\prime \prime} \mathrm{N}-75^{\circ} 44^{\prime} 14^{\prime \prime}$ y $75^{\circ} 52^{\prime} 38^{\prime \prime}$ W) forman parte de una extensa área marina protegida al suroccidente del Caribe sobre la plataforma continental (Fig. 1c, Fig. 1d) que se compone de un sistema de islas cuyo origen se relaciona con el desarrollo geomorfológico del continente, por lo que a diferencia de Providencia se han visto afectadas por los mismos procesos que modificaron las costas colombianas, como el diapirismo de lodo; la actividad tectónica de compresión y cizallamiento entre las placas Nazca, Suramericana y Caribe; y los cambios en el nivel del mar (Díaz et al., 2000; LópezVictoria \& Díaz, 2000; Posada et al., 2011). Su configuración actual ocurrió hace aproximadamente 2500 años y la circulación costera, el efecto de los ríos Sinú y Magdalena, el canal del Dique y la circulación de pequeñas corrientes son los elementos principales que modelan la dinámica del flujo costero entre las islas del Rosario y San Bernardo. Este sistema de islas se caracteriza por una topografía kárstica, que se originó cuando los arrecifes coralinos quedaron expuestos a los agentes atmosféricos durante los periodos glaciales del Pleistoceno y por los diapiros de lodo, que causan una alta tasa de sedimentación y la emanación de gases, razón por la que esta región se considera tectónicamente activa (Duque-Caro, 1980; Rangel \& Posada, 2005; Rangel \& Idarraga, 2010).

La circulación oceánica del área depende de la geografía de la región que obliga a las aguas de la corriente Caribe, que fluyen en dirección este, a recircular hacia el sur por efecto de la variación batimétrica provista por la elevación centroamericana, dando origen a un sistema a mesoescala de circulación anticiclónica que incluye el ciclón del Golfo de Mosquitos y el giro Panamá-Colombia, cuya su dinámica interanual afecta los patrones de conectividad del suroccidente del Caribe y junto con los vientos alisios y el desplazamiento anual de la Zona de Confluencia Intertropical son responsables de la surgencia de la Guajira (Andrade, 2001; Andrade et al., 2003, Lin et al., 2012).

Muestreo: Se realizaron muestreos mensuales desde septiembre 2011 hasta marzo 2012 con una intensidad de uno a tres días por sitio. La inspección se hizo en diferentes puntos de los arrecifes mediante buceo libre (tres buzos por muestreo), capturando manualmente los ejemplares vivos y ubicándolos en un reservorio con agua dentro de la lancha. Se registraron las coordenadas de captura, el sexo y el estado de madurez sexual. Los jóvenes se identificaron por presentar el abdomen sellado por dos 
protuberancias que encajan, a manera de llave y cerradura, con una muesca que se encuentra en los esternitos torácicos; mientras que los adultos maduros se identificaron mediante el grado de desarrollo de caracteres sexuales secundarios: en los machos se presenta mayor desarrollo del abdomen y robustecimiento de los quelípedos y en las hembras el abdomen se torna ancho y redondeado; adicionalmente se separó en otra categoría a las hembras que portaban la masa de huevos. Los individuos fueron fotografiados con ayuda de un soporte diseñado para tal fin, que consta de una base móvil con amarras de sujeción y una escala para ubicarlos en el encuadre correcto, manteniendo la cámara siempre a una distancia focal de $45 \mathrm{~cm}$ del objetivo. Posteriormente los individuos fueron liberados al medio.

Análisis: Los análisis se realizaron con el programa de libre distribución "Collecting Landmarks for Identification and Characterization" (CLIC) en: mome-clic.com (Dujardin, 2012). Se seleccionaron solo los individuos maduros, 276 en total: 103 hembras ovadas y 173 machos (Cuadro 1). Los puntos anatómicos de referencia (en inglés, landmarks) se marcaron solo en la mitad derecha del caparazón, para evitar la redundancia debida a la simetría (Cadrin, 2000) y se ubicaron en lugares que delimitaban los órganos internos o resumían la geometría del caparazón (Fig. 2), según lo propuesto por Bermúdez (2007) para crustáceos (Fig. 2).

Variables morfogeométricas: Se eligieron 12 landmarks (Bookstein, 1991), 10 tipo I que representan la yuxtaposición de diferentes tejidos o centros de pequeñas inclusiones (puntos 1, 2, 5-12); un landmark tipo II que representa el punto de máxima curvatura del lóbulo epibranquial (punto 3) y un landmark tipo III que corresponde al ancho máximo del caparazón, proyectado a partir de la región cardiaca (punto 4; Fig. 2). Los landmarks digitalizados como coordenadas (x, y) y la escala, se usaron para calcular el centro geométrico o "centroide" Cs, el cual se utilizó como variable de tamaño. Adicionalmente sobre las coordenadas (x, y) digitalizadas, se aplicó un Análisis Generalizado de Procrustes, el cual es un algoritmo de superposición que elimina los componentes no biológicos de la forma: escala, orientación y posición; con el fin de obtener las deformaciones parciales de los individuos respecto al consenso "partial warps" PWs (Rohlf, 1990; Rohlf \& Slice, 1990). Las PWs se sometieron a Análisis de Componentes Principales para

CUADRO 1

Tendencia central y dispersión de los tamaños de los centroides de los machos y hembras de las islas de Providencia (Prov) Rosario (Ros) y San Bernardo (Ber)

TABLE 1

Descriptive statistics and normality distribution test of the carapace centroids sizes of males and females from Providence (Prov) Rosario (Ros) and San Bernardo (Ber) islands

\begin{tabular}{cccccc}
$\mathrm{N}$ & Media & Mediana & DS & \multicolumn{2}{c}{ Normalidad K-S } \\
56 & 13.363 & 13.421 & 0.872 & 0.086 & $>0.20 * *$ \\
54 & 14.821 & 15.087 & 1.175 & 0.107 & $<0.15$ \\
24 & 10.095 & 10.207 & 0.953 & 0.159 & $<0.15$ \\
76 & 11.587 & 11.458 & 1.295 & 0.078 & $>0.20 * *$ \\
23 & 9.205 & 9.217 & 0.844 & 0.192 & $<0.15$ \\
43 & 8.911 & 8.941 & 0.612 & 0.089 & $>0.20 * *$ \\
\hline
\end{tabular}

La prueba de normalidad de Kolmogorov-Smirnov incluye el estadístico d, el p valor $(\alpha$ 0.05) con corrección de Lilliefors.

** Valores significativos.

The Kolmogorov-Smirnov test includes the statistic d, p-value corrected by Lilliefors. ** Significative values. 


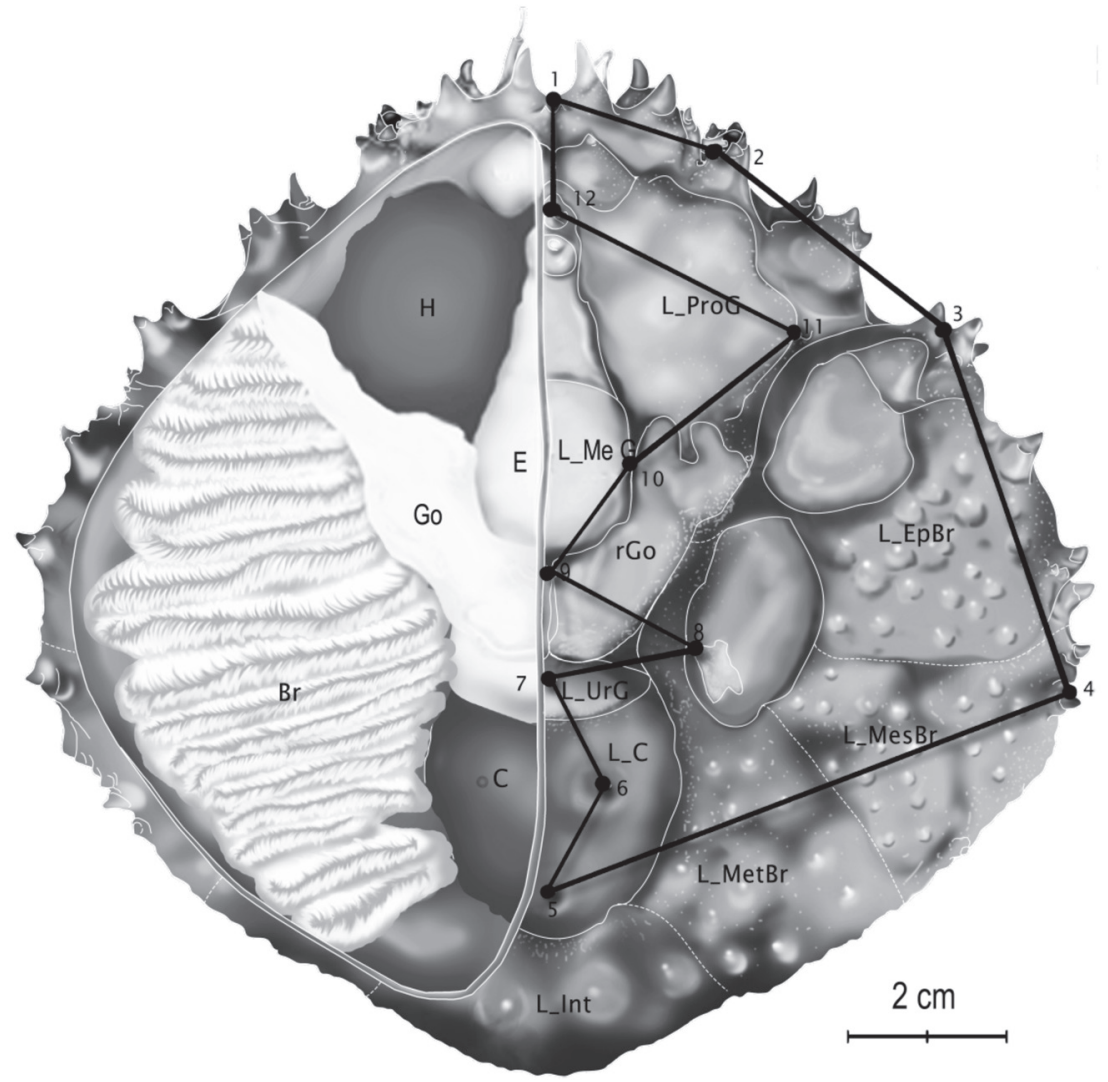

Fig. 2. Esquema de las regiones que conforman el caparazón con los 12 puntos anatómicos elegidos; al lado izquierdo se observan los principales órganos que se encuentran bajo las estructuras. Br: branquia; C: corazón; E: estómago; G: ganglio; Go: gónada; H: hepatopáncreas; L_C: lóbulo cardíaco; L_EpBr: lóbulo epibranquial; L_MesBr: lóbulo mesobranquial; L_MeG: lóbulo mesogástrico; L MetBr: lóbulo metabranquial; L_ProG: lóbulo protogástrico; L_UrG: lóbulo urogástrico; rGo: región gonadal. Basado en Rathbun (1925).

Fig. 2. Scheme of D. spinosissimus carapace showing 12 selected landmarks. On the left side are the most important organs under the carapace. Br: gill; C: heart; E: stomach; G: ganglion; Go: gonad; H: hepatopancreas; L_C: cardiac lobe; L_EpBr: epibranchial lobe; L_MesBr: mesobranchial lobe; L_MeG: mesogastric lobe; L MetBr: metabranchial lobe; L_ProG: protogastric lobe; L_UrG: urogastric lobe rGo: gonadal region. Based in Rathbun (1925).

producir las deformaciones relativas o "Relative wraps" RW (Dujardin, 2012), las cuales fueron utilizadas como variables de conformación. Para determinar la variación atribuible al error cometido al marcar los landmarks, se realizaron pruebas de repetibilidad (Harper, 1994; Arnqvist \& Mårtensson, 1998) sobre las variables obtenidas después de marcar dos veces los puntos sobre cada una de las 276 imágenes incluidas en este estudio: el tamaño centroide, las coordenadas alineadas después de la superposición de Procrustes y las variables RW.

Diferencias de tamaño: Sobre el tamaño de los centroides (Cs) se calculó el promedio, varianza y desviación estándar y se aplicaron pruebas de distribución normal de Kolmogorov-Smirnov sobre los residuales, y una prueba de Levene para determinar la homocedasticidad haciendo uso del programa STATISTICA 
StatSoft (2011). Debido a que la distribución no se ajustó a la normal, se realizaron comparaciones entre las medias sobre 10000 permutaciones, aplicando pruebas pareadas de Fisher con una corrección secuencial de Bonferroni en las comparaciones múltiples.

Diferencias de conformación: Se estimaron usando las deformaciones parciales o "Relative warps" (RW) obtenidas mediante Análisis de Componentes Principales. Los supuestos de normalidad y homocedasticidad multivariada se probaron con el estadístico Mardia (1970) para asimetría y Kurtosis; la prueba Doornik y Hansen (1994) y la prueba M-Box del programa PAST V.3 folk.uio.no/ohammer/past/ (Hamer, Harper, \& Ryan, 2013).

A partir de las RW se calcularon la conformación promedio entre sexos y entre orígenes geográficos, y las distancias Euclidianas entre las conformaciones promedio de los grupos comparados, las cuales se utilizaron para construir un árbol utilizando el algoritmo de Neighbour-Joining. La representación gráfica en forma de árbol resume en una sola imagen la variación total de la conformación, a diferencia de la representación cartesiana que se construye solo con dos componentes principales y no proporciona una representatividad completa.

Finalmente, la significancia estadística de las distancias Euclidianas entre sexos y entre orígenes geográficos se calculó mediante 10000 permutaciones y las comparaciones múltiples se ajustaron a la corrección secuencial de Bonferroni. Esta aproximación se utilizó en lugar de una prueba paramétrica debido a que no se cumplieron los supuestos de normalidad multivariada y homocedasticidad.

Efecto alométrico: Se realizó una regresión multivariada para estimar las relaciones de los rasgos durante el período de crecimiento (Huxley, 1932), entre cada componente principal (RW) y el tamaño del centroide (Cs). Cuando se encontró efecto alométrico, se utilizó la prueba MANCOVA (Análisis Multivariado de Covarianza) para determinar si las pendientes alométricas eran iguales y comparables.
Posteriormente, se eliminó el efecto residual del tamaño en las variables de conformación y se recalcularon las distancias euclidianas sobre 10000 permutaciones, ajustadas a corrección de Bonferroni.

\section{RESULTADOS}

La repetibilidad de las mediciones fue confiable en las coordenadas alineadas después de la superposición de Procrustes (>93\%); Cs (99.23\%) y en los 17 RW (91.86 - 98.26\%), indicando que las medidas son consistentes y la variación observada no está relacionada con el error del investigador.

Diferencias de tamaño: Los machos presentaron tamaños promedio de centroide mayores que las hembras. Entre regiones, la isla de Providencia presentó los mayores tamaños, seguido por islas del Rosario y San Bernardo, respectivamente; los supuestos de distribución normal no se cumplieron en los machos de Providencia, en las hembras de Rosario y en las hembras de San Bernardo (Cuadro 1, Fig. 3) y no se presentó homogeneidad entre las varianzas (Levene $3.1504 \mathrm{P}=0.009$ ) por lo que se usó estadística no paramétrica univariada para realizar las comparaciones.

Los tamaños de centroide, presentaron diferencias entre los grupos (Fisher Bonfer $0.003>\mathrm{P}=0.000$ ); excepto en los contrastes realizados entre las hembras y los machos de Rosario (Fisher $\mathrm{P}=0.022$ ) y las hembras de Rosario y San Bernardo (Fisher $\mathrm{P}=0.465$ ).

Diferencias de conformación y efecto alométrico: La distribución multivariada no se ajustó a la curva normal (Mardia: Asimetría 36.06 $\mathrm{P}=0.018$; Kurtosis 487.3 $\mathrm{P}=0.0006$ ) (Doornic \& Hansen 64.31 $\mathrm{P}=0.0087$ ), por lo cual se usó estadística no paramétrica para determinar las diferencias.

La regresión multivariada indicó un efecto alométrico $(\mathrm{P}=0.000)$ y se estableció que las pendientes alométricas eran comparables, con base en el análisis multifactorial de varianza ( $\lambda$ Wilks 0.623; F(100; 1199.9) 1.223 P=0.074). 


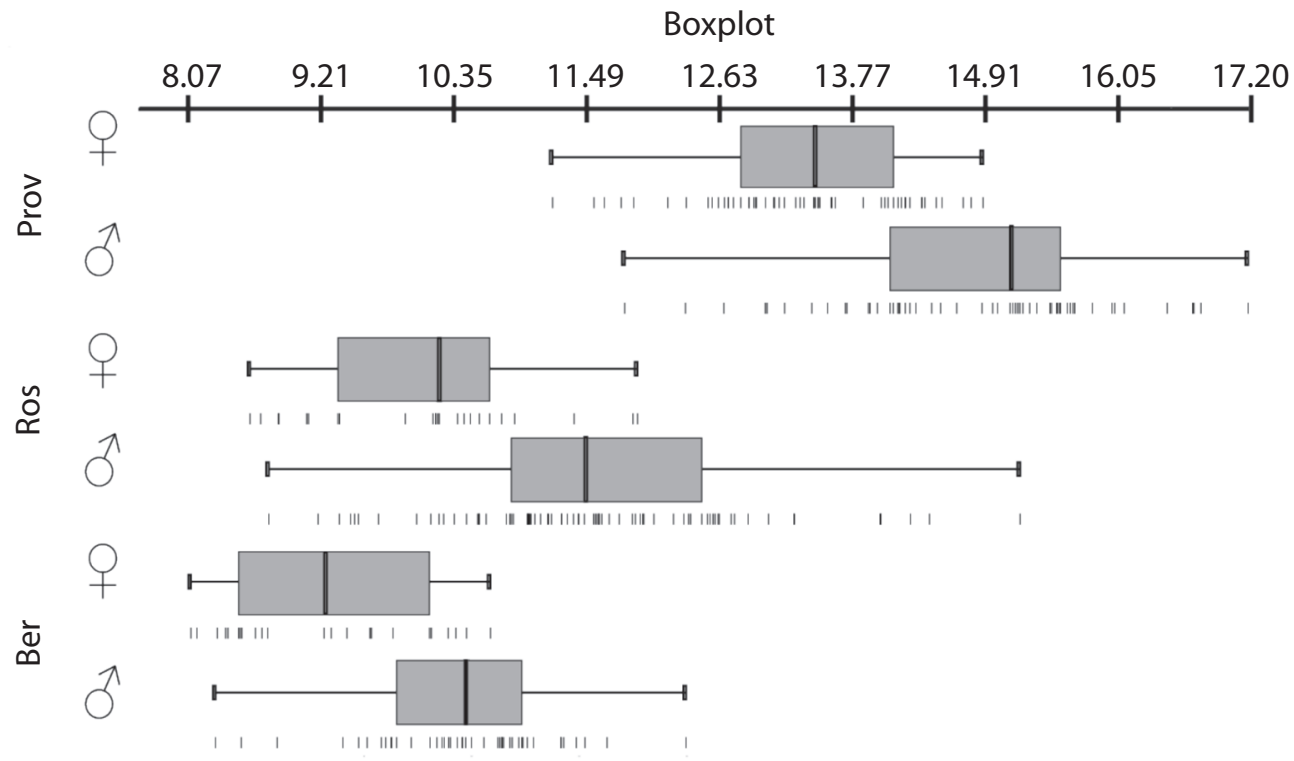

Fig. 3. Distribución de los tamaños centroides entre islas y sexos.

Fig. 3. Centroid size distribution among islands and between sexes.

En el árbol de distancias euclidianas (Fig. 4) se observan dos ramas principales separando a Providencia de Rosario y San Bernardo acorde con las distancias geográficas. La rama superior separa a los machos de Rosario; luego, se separan los machos de San Bernardo y finalmente, la menor distancia morfométrica, ocurre entre las hembras de Rosario y San Bernardo. Las distancias euclidianas después de corregir el efecto alométrico exhiben diferencias entre todas las agrupaciones $(0.000<\mathrm{P}<0.0003$ Bonfer; 10000 permutaciones) indicando que el sexo y la región afectan la conformación morfométrica de los caparazones.

Los análisis de machos y hembras por separado indican que existe efecto alométrico en ambos sexos ( $9 \mathrm{P}=0.000 ; \widehat{\partial}=0.000)$ las pendientes alométricas son comparables tanto en las hembras ( $\lambda$ Wilks $0.632 ; \mathrm{F}(40 ; 156)$ $1.004 \mathrm{P}=0.474)$ como en los machos $(\lambda \mathrm{Wilks}$ 0.792 ; $\mathrm{F}(40 ; 296) 0.792 ; \mathrm{P}=0.625)$ y las distancias euclidianas presentan la misma ordenación relacionada con las distancias geográficas observadas en el análisis en conjunto (Fig. 5).

\section{DISCUSIÓN}

Los resultados indican que existe variabilidad fenotípica tanto en el tamaño como en la conformación del caparazón de $D$. spinosissimus en función del sexo y procedencia geográfica. Tales variaciones pueden explicarse mediante causas netamente biológicas, ecológicas o por factores capaces de propiciar diferenciación genética. Aunque las diferencias morfológicas no permiten discernir entre una y otra causa, es por lo menos evidente que alguna está favoreciendo a la variación intraespecífica entre las regiones; razón por la cual, se discuten a continuación.

Los tamaños promedio de caparazón fueron mayores en Providencia que presenta una mayor influencia oceánica que las islas del Rosario y San Bernardo, cercanas al continente. Esta tendencia se ha observado en otros estudios realizados en el Caribe utilizando diferentes variables de tamaño (Munro, 1983; Guzmán \& Tewfik, 2004; Baeza, Anderson, Spadaro, \& Behringer, 2012). Por ejemplo, 


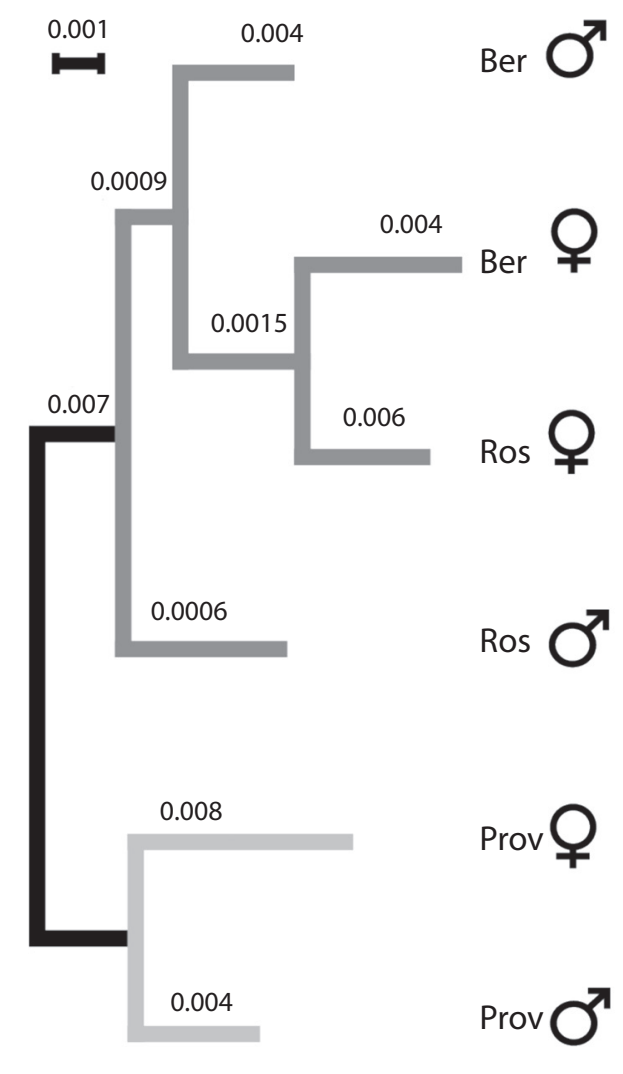

Fig. 4. Árbol Neighbour-Joining de las distancias euclidianas entre islas y sexos. Todas las distancias Euclidianas presentaron una significancia alta después de la corrección por tamaño $(0.000<\mathrm{P}<0.0003 ; 10000$ permutaciones). Ber: San Bernardo; Prov: Providencia;

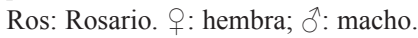

Fig. 4. Neighbour-Joinning tree based on Euclidian distances among islands and sexes. All Euclidian distances were highly significant after size correction $(0.000<\mathrm{P}<0.0003 ; 10000$ permutations $)$. Numbers in the branches indicate the bootstrap values. Ber: San Bernardo; Prov: Providence; Ros: Rosario. 우 female; $\hat{\jmath}$ : male.

las tallas registradas en Jamaica ( $q 12.28$ y ठ̂ $13.1 \mathrm{~cm}$; Munro, 1983) son similares a los tamaños obtenidos en Providencia ( $q$ 1 13.36 - $\widehat{\sigma}$ $14.82 \mathrm{~cm}$ ), mientras que la frecuencia modal de ejemplares registrados en Panamá ( +11 y $\delta$ 12.5 cm; Guzmán \& Tewfik, 2004) y las tallas promedio de individuos de la Florida ( $\widehat{c} 8$ - 9 cm y 97.12 - 8 cm; Baeza et al., 2012), son similares a los tamaños registrados en Rosario
( 10.10 y $\precsim$ đ $11.59 \mathrm{~cm}$ ) y San Bernardo (우 9.21 y ô $10.33 \mathrm{~cm}$ ).

Siguiendo a Wagner (1991) la tendencia a que en las islas oceánicas se presenten tallas más grandes que en el continente, puede resultar de una menor exposición de los animales a los cambios de salinidad, temperatura, contaminación y extracción. Estos cambios no necesariamente se relacionan con las condiciones ambientales a las que se encuentran sometidos los adultos; ya que en los crustáceos, los cambios más importantes del fenotipo debido a la variabilidad ambiental, ocurren durante las primeras etapas de vida, en tanto que son poco relevantes en la adultez (Giménez, 2006) y en el caso de Rosario y San Bernardo, las larvas pueden ser llevadas de las islas hacia los estuarios del continente, en los cuales la temperatura y la salinidad varía considerablemente. La posibilidad de que $D$. spinosissimus migre fue planteada por Munro (1983) quien no observa la presencia de juveniles de tallas pequeñas en los arrecifes $(<4 \mathrm{~cm})$; concordando con nuestras observaciones en campo.

En el suroccidente de la cuenca Colombia, los cambios de temperatura cerca al continente se deben al incremento del caudal de los ríos Magdalena y Sinú debido a las precipitaciones que ocurren entre junio-agosto y septiembrenoviembre, los cuales aumentan el aporte de aguas dulces, elevando la temperatura costera hasta los $30^{\circ} \mathrm{C}$ (Ruíz-Ochoa, 2011). Ya que durante las primeras etapas de muda, los crustáceos son más sensibles al estrés osmótico, y el efecto súbito de los cambios de temperatura sobre las larvas podría reducir la asimilación energética y la conversión de tejido para crecer (Anger, 2003); además, cuando aumenta la temperatura, la madurez se alcanza a edades más tempranas provocando una menor ganancia de tamaño (Hartnoll, 1982). Por esta razón, los individuos que habitan en las islas cercanas al continente podrían ser más susceptibles frente a alteraciones debidas a factores tales como descargas continentales, contaminación o el calentamiento global. Otros posibles factores que afectan las tallas en las islas del Rosario y San Bernardo, son la profundidad y 

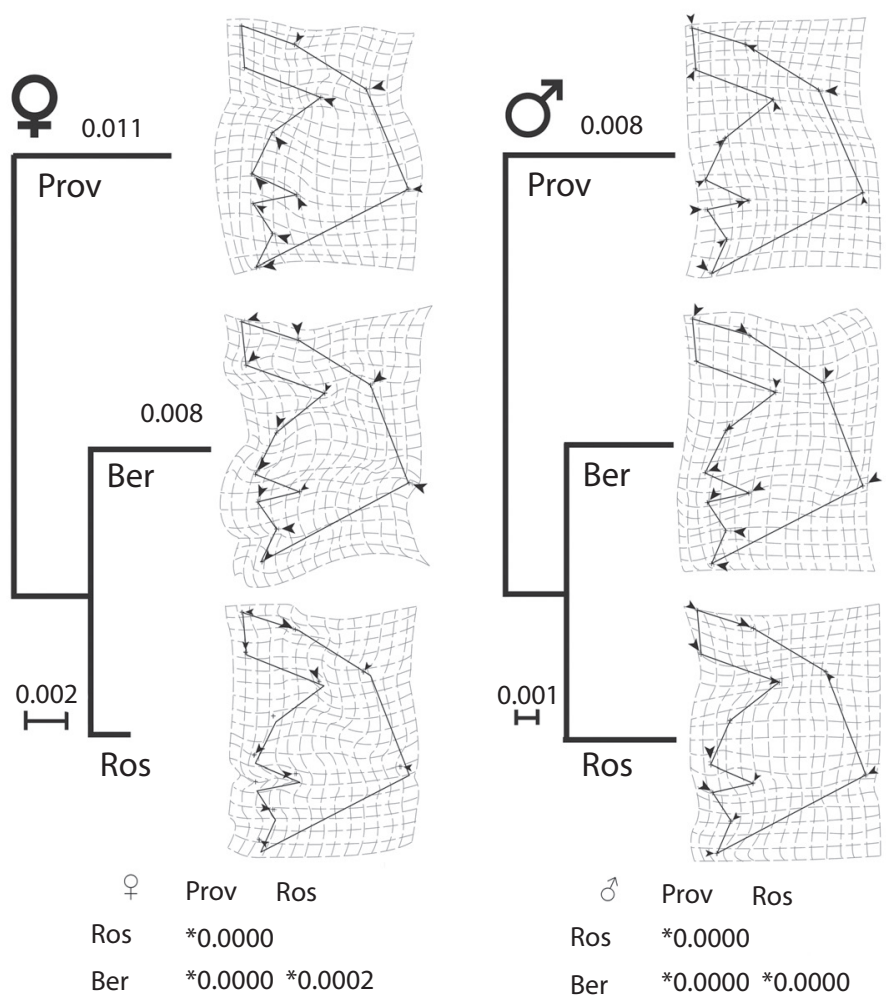

Fig. 5. Árbol Neighbour-Joining de las distancias euclidianas entre islas en hembras (†) y machos (ð̋) de D. spinosissimus. A la derecha están representas las rejillas de deformación mostrando la variación de la conformación con respecto a la forma consenso. Todas las distancias Euclidianas presentaron una significancia alta después de la corrección por tamaño $(0.000<\mathrm{P}<0.0002 ; 10000$ permutaciones $)$. Ber: San Bernardo; Prov: Providencia, Ros: Rosario.

Fig. 5. Neighbour-Joinning tree based on Euclidian distances among islands in females $(+)$ and males $\left(\circlearrowright^{\Uparrow}\right)$ of $D$. spinosissimus. On the right of each tree the deformation grids are represented showing the carapace shape variation in respect to consensus. All Euclidian distances were highly significant after size correction $(0.000<\mathrm{P}<0.0002 ; 10000$ permutations $)$. Numbers in the branches indicate the bootstrap values. Ber: San Bernardo; Prov: Providence, Ros: Rosario.

la disponibilidad de espacios crípticos, ya que el acceso es más fácil por la poca profundidad a la que se encuentran los corales y las formaciones con poca altura generan menos espacios crípticos para los adultos.

Las variaciones ambientales antes descritas también pueden explicar las diferencias de conformación del caparazón de D. spinosissimus entre islas. Además, los disturbios de tipo antropogénico, debido a las plumas de sedimentos del canal del Dique (Alvarado \& Corchuelo 1992), pueden tener un efecto negativo en las larvas, no solo por causar mortalidad (Pardo, Véliz, Wehrtmann, \& Klaus, 1997; Gebauer, Paschke \& Anger, 2003), sino porque pueden afectar el desarrollo ontogénico (Anger, 2003; Pechenic, 1999), o enviar señales químicas que favorezcan el auto-reclutamiento (Rodríguez, Ojeda, \& Inestrosa, 1992; Miller \& Morgan, 2013). La capacidad sensorial de las larvas de los crustáceos, permite que los individuos continuamente monitoreen las condiciones ambientales, lo cual puede estimular una respuesta rápida y continua frente a los cambios del medio. Como ocurre en otras especies, estos mecanismos pueden influir en el patrón corporal que desarrollará el individuo cuando ocurra la metamorfosis a su forma vida bentónica (Schlichting \& Smith, 2002), teniendo incluso consecuencias radicales sobre la 
forma y comportamiento de los adultos (Hartnoll, 1978; Anger, 1983; Pardo et al., 1997; Petriella \& Boshi, 1997; Haye, Salinas, Acuña, \& Poulin, 2010). Los cambios fenotípicos en respuesta a la heterogeneidad ambiental parecen ser un mecanismo generalizado en cangrejos ya que han sido descritos en otras especies como Carcinus maenas (Silva et al., 2010), Cyrtograpsus affinis (Lezcano et al., 2012) y Cyrtograpsus angulatus (Idaszkin et al., 2014).

Otra explicación posible es que las diferencias fenotípicas entre islas tengan una causa genética. La idea anterior está basada en los patrones de corrientes entre islas y las características de las larvas de D. spinosissimus. La baja interacción entre las islas debida a los patrones de corrientes es congruente con este supuesto, ya que las aguas que pasan por Providencia circulan hacia la cuenca Caimán o permanecen en el archipiélago mientras que la masa de agua del continente permanece recirculando en los giros ciclónicos (Andrade, 2000; 2001). Se ha demostrado que dichas corrientes son responsables del intercambio larval limitado que se observa entre varias regiones de Centroamérica y Colombia, presentando un porcentaje de conectividad menor a $0.05 \%$, el más bajo de todo el Caribe (Cowen et al., 2006). Bustamante y Paris (2008) sugieren incluso que Providencia hace parte de la unidad de conectividad "archipiélago colombiano y Jamaica", que es distinta a la unidad "continental de Colombia", a la que pertenecen las islas del Rosario y San Bernardo, con base en varios patrones de dispersión larval en peces y especies bentónicas como el caracol pala Strombus gigas (Paris, Pérez-Pérez, Kool, \& Aldana, 2008).

Si bien D. spinosissimus presenta ciclos de reproducción continua y puestas numerosas de aproximadamente 27000 huevos en Providencia y 14000 en Islas del Rosario (Pinzón, 2011), la distancia potencial de dispersión de la larva es baja, debido a que es lecitotrófica y solo permanece seis días en el plancton (Provenzano \& Bronwell, 1977). Por lo anterior, existe muy poca probabilidad de que las larvas que son arrastradas aguas afuera del ecosistema parental sobrevivan, pues el incremento en el consumo de energía metabólica afecta la capacidad de la muda a postlarva (Elkin \& Marshall, 2007).

Las diferencias de conformación del caparazón de los cangrejos entre las islas continentales de Rosario y San Bernardo son particularmente interesantes, ya que a pesar de ser un mosaico fragmentado, las islas tienen un desarrollo geomorfológico y paisajístico muy similar y se encuentran a una distancia relativamente corta de aproximadamente $30 \mathrm{~km}$ (Díaz et al. 2000; Cendales, Zea, \& Díaz, 2002). No obstante, la corta duración del ciclo larval de D. spinosissimus y la evidencia genética de que otras especies bentónicas como el caracol pala Strombus gigas (Márquez et al., 2013) y el coral Montastrea annularis (Sánchez, 2009), presentan una estructura poblacional entre Islas del Rosario y San Bernardo, apuntan a que puede existir un patrón que afecta la conectividad de la comunidad bentónica entre las dos islas, lo cual podría explicar las diferencias fenotípicas encontradas en nuestro estudio.

Aunque se conoce poco sobre la circulación a micro escala entre las islas del Rosario y San Bernardo, se deben tener en cuenta algunos aspectos locales que podrían afectar la conectividad entre las dos islas; por ejemplo, durante la estación húmeda se forma una célula ciclónica en las islas del Rosario debida al efecto del río Magdalena (INVEMAR, 2003); asimismo, a través de los caños Matunilla y Lequerica, existe una corriente de agua dulce proveniente del canal del Dique que atraviesa el parque entre las regiones Rosario - San Bernardo (Posada et al., 2011). Por otra parte, las terrazas kársticas y los domos diapríricos forman canales por los que circulan flujos pequeños de agua creando corrientes ascendentes (Cendales et al., 2002; Posada et al., 2011), que generan movimientos cerca del arrecife, haciendo que éstas se comporten como pequeñas piscinas en las que podrían quedar retenidas las larvas, que luego serían transportadas en dirección a la costa en lugar de ir a aguas abiertas.

En conclusión, este estudio encontró diferencias en el tamaño y conformación del 
caparazón de machos y hembras de D. spinosissimus entre tres islas, las cuales pueden ser concordantes con diferencias genéticas o plasticidad fenotípica dada la variación ambiental. Queda por contrastar esta hipótesis con marcadores genéticos polimórficos neutros que permitan determinar el grado de conectividad entre las islas y estimar el papel del ambiente en las variaciones fenotípicas observadas. También quedan por caracterizar variables ambientales, dinámicas de corrientes a pequeña escala y el efecto potencial de las actividades antropogénicas sobre la variación fenotípica de este cangrejo del Caribe.

\section{AGRADECIMIENTOS}

Agradecemos a Colciencias y a la Universidad Nacional de Colombia, Sedes Caribe y Medellín, por la financiación de este trabajo en el marco del proyecto 1361-521-28198 titulado "Evaluación del estado de las poblaciones del cangrejo rey del caribe, Mithrax spinosissimus (Lamarck, 1818), un modelo para el estudio de invertebrados bentónicos amenazados en Colombia". Expresamos nuestros agradecimientos a la Unidad de Parques Nacionales y Naturales, en especial a la directora del McBean Old Providence Marcela Cano y a Esteban Zarza en el Parque Nacional Natural Islas del Rosario y San Bernardo. Contribución No. 420 del Instituto de Estudios en Ciencias del Mar del Sede Caribe de la Universidad Nacional de Colombia.

\section{RESUMEN}

El cangrejo rey del Caribe Damithrax spinosissimus es un recurso pesquero catalogado en estado de vulnerabilidad y de cuya biología se conoce muy poco. Su distribución agregada en mosaico y la gran heterogeneidad ambiental dada por las características oceanográficas, paisajísticas y ecológicas del Caribe, pueden propiciar a que exista variabilidad fenotípica en función de la procedencia geográfica de los individuos. Por esta razón, el objetivo de esta investigación fue determinar si existe variabilidad morfogeométrica en los caparazones de ejemplares procedentes de tres islas del Caribe suroccidental: Providencia, Rosario y San Bernardo. La primera de ellas con una influencia netamente oceánica y las dos siguientes, cercanas al continente, con mayor influencia de las dinámicas costeras. Para ello se capturaron y fotografiaron 276 individuos: 103 hembras ovadas y 173 machos, sobre los cuales se realizaron análisis morfogeométricos, tomando 12 puntos anatómicos de referencia (Landmarks) en una de las mitades del caparazón. Las diferencias de tamaño entre sexos y entre orígenes geográficos se contrastaron mediante comparaciones pareadas de Fisher, el efecto alométrico se estimó mediante Análisis de Regresión Multivariada y el modelo de pendientes alométricas mediante Análisis Multivariado de Covarianza. La conformación promedio entre sexos y entre orígenes geográficos se computó de las deformaciones relativas, obtenidas mediante análisis de componentes principales; asimismo, las distancias Euclidianas entre las conformaciones promedio se utilizaron para construir un árbol con base en el algoritmo de Neighbour- Joining con una significancia calculada sobre 10000 permutaciones. Los resultados mostraron diferencias en el tamaño y conformación del caparazón entre sexos y entre las tres islas, siendo más evidentes las diferencias en Providencia. Estos resultados pueden ser explicados de manera no excluyente por diferencias genéticas y plasticidad fenotípica debida a la heterogeneidad ambiental del sector. Este estudio, primero en su clase, es un aporte al conocimiento de la especie y de él se concluye que deberían existir estrategias de manejo diferentes para las tres islas.

Palabras clave: morfometría geométrica, plasticidad fenotípica, especie vulnerable, Caribe suroccidental, Crustáceos.

\section{REFERENCIAS}

Alvarado, E. M., \& Corchuelo, M. C. (1992). Los nutrientes, la temperatura y la salinidad provenientes del canal del Dique como factores de deterioro en el Parque Nacional Natural Corales del Rosario (Cartagena, Colombia). En Senalmar. Memorias. Santa Marta, Colombia. 277-287.

Andrade, C. A. (2000). The Circulation and Variability of the Colombian Basin in the Caribbean Sea (Doctoral thesis ). University of Wales, Wales.

Andrade, C. A. (2001). Las corrientes superficiales en la cuenca de Colombia observadas con boyas de deriva. Revista de la Academia Colombiana de Ciencias, 96, 321-335.

Andrade, C. A., Barton, E. D., \& Mooers, N. K. (2003). Evidence for an Eastward flow along the Central and South American Caribbean Coast. Journal of Geophysical Research, 108(C6), 1-11.

Anger, K. (1983). Temperature and the larval development of Hyas araneus L. (Decapoda: Majidae); extrapolation of laboratory data to field conditions. Journal of Experimental Marine Biology and Ecology, 69, 203-215. 
Anger, K. (2003). Salinity as a key parameter in the larval biology of decapods crustaceans. Invertebrate Reproduction and Development, 43(1), 29-45.

Arnqvist, G., \& Mårtensson, T. (1998). Measurement error in geometric morphometrics: empirical strategies to assess and reduce its impact on measures of shape. Acta Zoologica, 44, 73-96.

Baeza, J. A., Anderson, J. R., Spadaro, A. J., \& Behringer, D. C. (2012). Sexual dimorphism, allometry, and size at first maturity of the Caribbean king crab, Mithrax spinosissimus, in the Florida Keys. Journal of Shellfish Research, 31(4), 909-916.

Bermúdez, A. (2007). Populationsstruktur und genetische Isolierung der amerikanischen Springkrebs-Arten Munida flinti, Agononida longipes und Munidopsis riveroi (Crustacea, Decapoda, Anomura, Galatheidae). Fachbereich 08 Biologie, Chemie und Geowissenschaften. Gießen: Universität Gießen.

Bermúdez, A., Campos, N. H., \& Navas, G. R. (2002). Mithrax spinosissimus. En N. Ardila, G. R. Navas, \& J. Reyes (Eds.), Libro Rojo de los Invertebrados Marinos de Colombia (pp.116-117). Colombia: INVEMAR. Ministerio de Medio Ambiente. La Serie Libros rojos de Especies Amenazadas de Colombia Bogotá, Colombia.

Boltovskoy, D. (1981). Atlas del zooplancton del Atlántico sudoccidental y métodos de trabajo con el zooplancton marino. Argentina: Publicación especial del Instituto Nacional de Investigación y Desarrollo Pesquero INIDEP, Mar del Plata Argentina.

Bookstein, F. L. (1991). Morphometric tools for landmark data. New York: Cambridge University Press.

Bustamante, G., \& Paris, C. B. (2008). Marine population connectivity and its potential use for the nomination of new World Heritage Sites in the wider Caribbean. En R. Grober-Dunsmore and B. D. Keller (Eds.), Caribbean connectivity: Implications for marine protected area management (p. 195). Proceedings of a Special Symposium, 9-11. Nov. 2006, 59th. Annual meeting Gulf and Caribbean Fishering Institute, Marine Sanctuaries Conservation Series. Department of Commerce, National Oceanic and Atmospheric Administration, Office of National Marine Sanctuaries, Silver Spring, MD. Belize City, Belize.

Cadrin, S. X. (2000). Advances in morphometric identification of fishery stocks. Reviews in Fish Biology and Fisheries, 10, 91-112.

Cendales, M. H., Zea, S. E., \& Díaz, J. M. (2002). Geomorfología y unidades ecológicas del complejo de arrecifes de las Islas Rosario e isla Barú (Mar Caribe, Colombia). Revista de la Academia Colombiana de Ciencias, 26(101), 497-510.

CORALINA-INVEMAR. (2012). Gómez López, D. I., Segura-Quintero, C., Sierra-Correa, P. C., \&
Garay-Tinoco, J. (Eds.), Atlas de la Reserva de Biosfera Seaflower. Archipiélago de San Andrés, Providencia y Santa Catalina (p. 180). Instituto de Investigaciones Marinas Costeras "José Benito vives de Andreis"-INVEMAR- y Corporación para el Desarrollo Sostenible del Archipiélago de San Andrés Providencia y Santa Catalina -CORALINA-. Serie de Publicaciones Especiales de INVEMAR. Santa Marta, Colombia.

Cowen, R. K., Paris, C. B., \& Srinivasan, A. (2006). Scaling of Connectivity in Marine Populations. Science, 311, 511-527.

Díaz, J. M., Barrios, L. M., Cendales, M. H., GarzónFerreira, J., Geister, J., López-Victoria, M., \& Zea, S. (2000). Areas coralinas de Colombia. INVEMAR, Serie Publicaciones Especiales (176 p). Santa Marta. Colombia.

Doornik, J. A., \& Hansen, H. (1994). An omnibus test for univariate and multivariate normality. En Nuffield Economics Working Papers.

Duque-Caro, H. (1980). Geotectónica y evolución de la región noroccidental colombiana. Boletin Geológico de Ingeominas (p. 23-37). Bogotá, Colombia.

Dujardin, J. P. (2012). Click package for Windows. Institut de Recherches pour le Dévelopement (IRD. France). Retrieved from http://www.mpl.ird.fr/morphometrics.

Elkin, C., \& Marshall, D. J. (2007). Desperate larvae: influence of deferred costs and habitat requirements on habitat selection. Marine Ecology Progress Series, $335,143-153$.

Felder, D. L., Álvarez, F., Goy, J. W., \& Lemaitre, R. (2009). Decapoda (Crustacea) of the Gulf of Mexico, with Comments on the Amphionidacea. En D. L. Felder \& D. K. Camp (Eds.), Gulf of Mexico-Origins, Waters, and Biota. Biodiversity (pp. 1019-1104). Texas: Texas A\&M Press, College Station, Texas.

Gebauer, P., Paschke, K., \& Anger, K. (2003). Delayed metamorphosis in decapod crustaceans: evidence and consequences. Revista Chilena de Historia Natural, $76,169-175$

Giménez, L. (2006). Phenotypic links in complex life cycles: conclusions from studies with decapods crustaceans. Integrative and Comparative Biology, 45(5), 615-622.

Guzmán, H., \& Tewfik, A. (2004). Population caracteristics and co-ocurrence of three exploited decapods (Рanulirus argus and Mithrax spinosissimus) in Bocas del Toro, Panamá. Journal of Shellfish Research, 23(2), 575-580.

Hammer, Ø., Harper, D. A. T., \& Ryan, P. D. (2013). PAST: Paleontological Statistics Software Package for Education and Data Analysis. Palaeontología Electronica, 4(1), 9. 
Harper, D. G. C. (1994). Some comments on the repeatability of measurements. Ringing \& Migration, 15, 84-90.

Hartnoll, R. G. (1978). The determination of relative growth in Crustacea. Crustaceana, 34(3), 281-293.

Hartnoll, R. G. (1982). Growth. En D. E. Bliss \& L. G. Abele (Eds.), The Biology of Crustacea, 2, Embryology, Morphology and Genetics (pp. 111-196). New York: Academic Press.

Haye, P. A., Salinas, P., Acuña, E., \& Poulin, E. (2010). Heterochronic phenotypic plasticity with lack of genetic differentiation in the Southeastern Pacific squat lobster Pleuroncodes monodon. Evolution and Development, 12(6), 628-624.

Hiller, A., Kraus, H., Almon, M., \& Werding, B. (2006). Species boundaries based on color pattern, morphology and molecules, and evolutionary interrelationships between this complex and other Porcellanidae (Crustacea: Decapoda: Anomura). Molecular Phylogenetics and Evolution, 40, 547-569.

Huxley, J. S. (1932). Problems of relative growth. Baltimore: Johns Hopkins University Press.

Idaszkin, Y., Márquez, F., \& Nocera, A. (2014). Habitatspecific shape variation in the carapace of the crab Cyrtograpsus angulatus. Journal of Zoology, 290(2), 117-126.

INVEMAR. (2003). Programa Holandés de Asistencia para Estudios en Cambio Climático: Colombia. Definición de los estados de vulnerabilidad de los sistemas bio-geofisicos y socioeconómicos debido a un cambio en el nivel del mar en la zona costera colombiana (Caribe insular y Pacifico) y medidas para su adaptación (Informe técnico $\mathrm{N}^{\circ} 2$, p. 571). Caracterización e inventario. Santa Marta, Colombia.

Lamarck, J. B. P. A. de. (1818). Histoire naturelle des animaux sans vertèbres, présentant les caratères généraux et particuliers de cex animaux, leur distribution, leur casses, leur familles, leur genres et la citation des principales espèces qui s'y raportent précédé d' une introduccion offrant la détermination des caractères essentiels de l'animal sa distinction $d u$ végétal et des autres corps naturels, enfin l'exposition des principes fondamentaux de la zoologie. Deuxième edition, 5, 1-611.

Ledesma, F. M., Van der Molen, S., \& Barón, P. J. (2010). Sex identification of Carcinus maenas by analysis of carapace geometrical morphometry. Journal of Sea Research, 63, 213-216.

Lezcano, A. H., González-José, R., Spivak, E. D., \& Dellatorre, F. G. (2012). Geographic differences in the carapace shape of the crab Cyrtograpsus affinis (Decapoda: Varunidae) and its taxonomic implications. Scientia Marina, 76, 329-337.
Lin, Y., Sheng, J., \& Greatbatch, R. J. (2012). A numerical study of the circulation and monthly to seasonal variability in the Caribbean Sea: the role of Caribbean eddies. Ocean Dynamics, 62, 193-211.

López-Victoria, M. \& Díaz J. M. (2000). Morfología y estructuras de las formaciones coralinas del Archipiélago de Rosario y San Bernardo, Caribe colombiano. Revista de la Academia Colombiana de Ciencias, 24(91), 219-230.

Mardia, K. V. (1970). Measures of multivariate skewness and kurtosis with applications. Biometrika, 36, 519-530.

Márquez, E., Landínez-García, R. M., Ospína-Guerrero, S., Segura, J. A., Prada, M., Castro, E., Correa, J. L., \& Borda, C. (2013). Análisis Genético de Caracol Pala Strombus gigas del Caribe Suroccidental. 65 th Proceedings of Gulf and Caribbean Fisheries Institute (pp. 410-416).

Martínez-Campos, B., Campos, N. H., \& Bermúdez, A. (2012). Distribución de cangrejos ermitaños (Anomura: Paguroidea) en el mar Caribe colombiano. Revista de Biología Tropical, 60(1), 233-252.

Miller, S. H., \& Morgan, S. G. (2013). Phenotypic plasticity in larval swimming behavior in estuarine and coastal crab populations. Journal of Experimental Marine Biology and Ecology, 449, 45-50.

Munro, J. L. (Ed.). (1983). Caribbean Coral Reefs Fisheries Resourses. ICLARM studies and reviews. Manila, Filipinas: International center for living acuatic resourses management.

Pardo, L. M., Véliz, D., Wehrtmann, I. S., \& Klaus, A. (1997). Efecto de períodos de ayuno en el crecimiento de la zoea I de Petrolisthes violaceus (Guérin, 1831) (Decapoda: Anomura: Porcellanidae). Investigaciones Marinas, 25, 255-262.

Paris, C. B., Pérez-Pérez, M., Kool, J., \& Aldana Aranda, D. (2008). Segregation of Queen Conch, Strombus gigas, Populations from the Yucatan Peninsula, Mexico. En R. Grober-Dunsmore, \& B. D. Keller (Eds.), Caribbean connectivity: Implications for marine protected area management (p. 195). Proceedings of a Special Symposium, 9-11. Nov. 2006, 59 ${ }^{\text {th }}$ Annual Meeting Gulf and Caribbean Fishering Institute, Marine Sanctuaries Conservation Series. Department of Commerce, National Oceanic and Atmospheric Administration, Office of National Marine Sanctuaries, Silver Spring, MD. Belize City, Belize.

Pechenick, J. A. (1999). Of the advantages and disadvantages of larval stages in benthic marine invertebrate life cycles. Marine Ecology Progress Series, 177, 269-297.

Petriella, A. M., \& Boshi, E. E. (1997). Crecimiento en crustáceos decápodos: resultados de investigaciones 
realizadas en Argentina. Investigaciones Marinas Valparaiso, 25, 135-157.

Pinzón, M. (2011). Aspectos de la biología de Mithrax spinosissimus (Lamark, 1818) (Brachyura: Mithracidae) en el Caribe colombiano (Trabajo de grado). Universidad Nacional de Colombia, Bogotá, Colombia.

Posada, B. O., Morales, D., \& Henao, W. (2011). Diagnóstico de la erosión costera del territorio insular colombiano. INVEMAR, Serie Publicaciones Especiales 24 (p. 112). Santa Marta, Colombia.

Provenzano, A. J. Jr., \& Brownell, W. N. (1977). Larval and early-post larval stages of the West Indian spider crab, Mithrax spinosissimus (Lamark) (Decapoda: Majidae). Proceedings of the Biological Society of Washington, 90(3), 735-752.

Rangel, N., \& Idarraga, J. (2010). Geología general, morfología submarina y fasíes sedimentarias en el margen continental y los fondos oceánicos del mar Caribe colombiano. En: Biodiversidad del margen continental del Caribe colombiano. INVEMAR, Serie Publicaciones Especiales 24 (pp. 34-50). Santa Marta, Colombia.

Rangel, N., \& Posada, B. (2005). Geomorphology and erosive processes in the north coast of Cordoba department, Colombian Caribbean (Sector Paso Nuevo-Cristo Rey). Boletín de Investigaciones Marinas y Costeras - INVEMAR, 34, 101-119.

Rathbun, M. J. (1925). The spider crabs of America (613 p. +283 plates). Washington D.C.: Bulletin of Smithsonian Institute. Washington government printing office.

Rodríguez, S. R., Ojeda F. P., \& Inestrosa, N. C. (1992). Inductores químicos del asentamiento de invertebrados marinos bentónicos: importancia y necesidad de su estudio en Chile. Revista Chilena de Historia Natural, 65, 297-310.

Rohlf, F. J. (1990). Rotational fit (Procrustes) methods. En F. J. Rohlf, \& F. L. Bookstein (Eds.), Proceedings of the Michigan Morphometrics Workshop (pp. 227. 236). Michigan: Museum of Zoology, Ann Arbor, University of Michigan.

Rohlf, F. J., \& Slice, D. E. (1990). Extensions of the Procrustes method for the optimal superimposition of landmarks. Systematic Zoology, 39, 40-59.

Rufino, M., Abelló, P., \& Yule, A. B. (2004). Male and female carapace shape differences in Liocarcinus depurator (Decapoda, Brachyura): An application of geometric morphometric analysis to crustaceans. Italian Journal of Zoology, 71, 79-83.

Ruíz-Ochoa, M. A. (2011). Variabilidad de la Cuenca Colombia (Mar Caribe) asociada con El NiñoOscilación del Sur, vientos Alisios y procesos locales (Tesis Doctorado). Universidad Nacional de Colombia, Medellín, Colombia.

Sánchez, J. A. (2009). Diversidad genotípica, estructura y flujo genético en las poblaciones del coral Montastraea annularis (PNN Corales del Rosario y San Bernardo): un acercamiento mediante el uso de microsatélites (pp. 35-37). En Ministerio de Ambiente Vivienda y Desarrollo Territorial; Parques Nacionales Naturales de Colombia; Fondo Patrimonio Natural; Corporación Autónoma Regional de Cundinamarca - CAR. Primer Congreso de Áreas Protegidas. Hacia un Sistema Nacional de Áreas Protegidas Completo, Representativo y Eficazmente Gestionado. Bogotá, Colombia.

Schlichting, C. D., \& Smith, H. (2002). Phenotypic plasticity: linking molecular mechanism with evolutionary outcomes. Evolutionary Ecology, 16, 189-211.

Silva, I. C., Alves, M. J., Paula, J., \& Hawkins, S. J. (2010). Population differentiation of the shore crab Carcinus maenas (Brachyura: Portunidae) on the Southwest English coast based on genetic and morphometric analyses. Scientia Marina, 74, 435-444.

StatSoft. (2011). STATISTICA (Data analysis sofware system). Version 10. Recuperado de www.statsoft.com

Trevisan, A., Zanetti-Marochi, M., Costa, M., Santos, S., \& Masunari, S. (2012). Sexual dimorphism in Aegla marginata (Decapoda: Anomura). Nauplius, 20, 75-86.

Wagner, H. P. (1991). The genera Mithrax Latreille, (1818) and Mithraculus White, (1847) (Crustacea: Brachyura: Majidae) in the western Atlantic Ocean. Zoologische Verhandelingen, 264, 3-65.

Williams, A. (1984). Shrimps, lobsters, and crabs of the Atlantic coast of the Eastern United States, Marine to Florida. Washington D.C.: Smithsonian Institution Press.

Windsor, A. M., \& Felder, D. L. (2014). Molecular phylogenetics and taxonomic reanalysis of the family Mithracidae MacLeay (Decapoda: Brachyura: Majoidea). Invertebrate Systematic, 28, 145-173. 
